\title{
Exploiter un travail de corpus sur la place de l'épithète pour élaborer une progression didactique en FLE
}

\author{
Marie-Armelle Camussi-Ni, Annick Coatéval (Rennes) et Juliette Thuilier (Toulouse)
}

\begin{abstract}
This paper sets out the convergence between two approaches - a theoretical one and an applied one - aiming to tackle the problem of attributive adjective position in French. We show how the results of a corpus study contribute to the understanding of the issues faced by traditional descriptions designed for learners of French as a foreign language. This reflection leads us to create a learning progression that aims to give learners the keys to the linguistic skills that they lack. Even if the objectives of researchers in corpus linguistics and researchers in education are different, we think that the gap between usage and grammar requires learning sequences to be drawn up on the basis of current works based on corpora. This approach allows us to avoid learning sequences that either are unrealistic simplifications or necessarily non-exhaustive inventories.
\end{abstract}

\section{$1 \quad$ Introduction}

La multiplicité des travaux qui traitent de la disposition de l'adjectif épithète atteste des difficultés des linguistes pour en élaborer une description cohérente. Cette question n'est pas nouvelle - Erwin Reiner (1968) la fait remonter au $16^{\text {ème }}$ siècle -, elle continue à faire débat comme en témoignent les nombreux travaux qui lui ont été consacrés (cf. Forsgren 1978; Wilmet 1981; Delbecque 1990; Nølke 1996; Noailly 1999; Abeillé/Godard 1999...), et semble inépuisable quand on prend en compte les travaux actuels sur le sujet (cf. Benzitoun 2013; Thuilier et al. 2012 parmi d'autres).

Si cette question est problématique pour le linguiste, elle l'est aussi pour le didacticien en FLE qui doit anticiper les difficultés que vont rencontrer les apprenants, difficultés liées :

- aux calques de langue qui expliquent la production d'énoncés tels que ?le britannique accent $^{1}$

- à des généralisations hâtives qui peuvent entraîner la production de séquences comme ?du fin sel à partir de du gros sel ;

- ou encore à une incompréhension déstabilisante, quand les apprenants confrontent, par exemple, les énoncés la prochaine fois et ?la prochaine semaine.

\footnotetext{
${ }^{1}$ Le symbole ? désigne non pas un doute sur la grammaticalité de l'énoncé mais un énoncé de probabilité de réalisation faible.
}

Linguistik online 78, 4/16 - http://dx.doi.org/10.13092/lo.78.2949

CC by 3.0 
L'originalité de cet article tient à la convergence entre deux démarches portant sur la disposition de l'adjectif :

- celle d'une thèse (cf. Thuilier 2012), réalisée à partir d'une recherche sur le corpus French TreeBank, qui soutient que «la position de l'adjectif ne répond pas à des contraintes catégoriques imposant une position spécifique, mais à des contraintes qui favorisent une position plutôt que l'autre " (Thuilier 2012 : 5). L'auteur donne un statut à ces contraintes par le recours à la notion de contraintes préférentielles : "notre objectif sera de montrer que l'approche en termes de contraintes préférentielles est adaptée pour ce phénomène dans la mesure où elle permet de rendre compte de son caractère multidimensionnel » (Thuilier 2012: 5).

- et celle de travaux de didacticiens (cf. Camussi-Ni/Coatéval 2013) qui, confrontés à la récurrence des énoncés problématiques dans les corpus d'énoncés d'apprenants étrangers et aux impasses des descriptions des grammaires qui leur sont destinées, cherchent à substituer aux généralisations pédagogiques une compréhension et une maîtrise progressive des paramètres en jeu dans la disposition de l'épithète.

C'est ainsi que cet article fait dialoguer ces deux approches : (1) l'approche du chercheur qui a opté pour l'analyse de corpus afin de rendre compte non seulement " de l'influence » des différents facteurs (fréquence, longueur, morphologie...) qui entrent en jeu dans l'ordre des termes mais encore de leurs effets combinés ; (2) et l'approche des didacticiens confrontés au problème de l'enseignement de l'épithète en français et tenus par la nécessité de rendre compte de l'usage et d'en assurer une description cohérente qui compense le caractère parcellaire de l'usage chez les apprenants allophones.

Nous nous proposons, dans un premier temps, de confronter l'analyse des difficultés qui se posent à l'apprenant FLE aux descriptions présentées dans les manuels de grammaire. Par la suite, nous montrerons en quoi les résultats des travaux sur corpus de J. Thuilier permettent, sinon de résoudre les problèmes, du moins de les poser différemment en évitant les impasses des descriptions classiques. Nous verrons enfin comment le didacticien peut tirer profit et de la démarche d'analyse des corpus et des conclusions apportées afin de proposer des progressions qui s'appuient sur la réalité de la langue tout en prenant en compte une grammaire que l'apprenant se construit.

\section{Le problème de la position de l'adjectif pour un apprenant FLE}

La disposition de l'épithète constitue un casse-tête pour un apprenant étranger dans la mesure où ce dernier doit intégrer des règles d'usage qui obligent à considérer qu'il existe une certaine liberté dans ce phénomène mais que cette liberté n'est pas totale et qu'elle est contrainte par l'existence de « règles » non systématiques.

\subsection{Accepter la possibilité d'alternance de position}

La possibilité d'alternance peut être en soi une difficulté pour les apprenants étrangers, particulièrement si les langues qu'ils maîtrisent déjà présentent une contrainte de disposition de l'épithète, comme par exemple l'anglais ou le chinois qui placent systématiquement l'adjectif avant le nom. Ces apprenants courent le risque de nier la possibilité d'alternance et 
Exploiter un travail de corpus sur la place de l'épithète pour élaborer une progression didactique en FLE

d'opter pour une disposition fixe, par calque exact comme en (1) ou par calque inversé, comme en (2), s'ils repèrent une disposition inverse et la généralisent.

1) ?un normal film ${ }^{2}$

2) des cases petites $^{3}$

Le calque peut se voir confirmer dès leurs premiers contacts avec le français. Ainsi pourraient-ils être enclins à voir l'antéposition comme la règle générale s'ils se basent sur les quelques adjectifs très fréquents antéposés comme petit, grand, jeune, vieux ou beau. À partir des séquences du type une petite voiture, une grande voiture, une belle voiture, ils généraliseraient et produiraient?une familiale voiture, ?une rouge voiture, ?une révolutionnaire voiture... Ou bien, au contraire, dans la mesure où la postposition est la position la plus fréquente si l'on considère la classe entière des adjectifs (plus de $70 \%$ de postposition d'après les données de Thuilier (2012)), les apprenants pourraient faire de cette position la norme. Ainsi, produiraient-ils des énoncés tels que ?une voiture grosse ou ?une voiture vieille à partir de l'observation de séquences du type une voiture économique.

\subsection{Admettre que les possibilités d'alternance sont limitées}

Une fois admise la possibilité d'alternance de disposition, un autre risque se profile pour l'apprenant : celui de croire que cette liberté est totale, qu'il peut choisir l'ordre relatif du nom et de l'adjectif épithète, dans la mesure où il rencontre des adjectifs antéposés ou postposés (exemples 3 à 6) et qu'un même adjectif peut voir sa position alterner dans le syntagme (exemple 7)

3) une grande voiture

4) une voiture familiale

5) une belle voiture

6) une voiture récente

7) une splendide voiture / une voiture splendide

L'apprenant ignore alors que l'alternance totalement libre comme dans l'exemple (7) est loin d'être un cas général. Croire que la liberté de disposition est totale peut conduire à des productions où la disposition alterne de façon totalement libre, comme dans l'énoncé suivant :

8) ?le taux des originales informations sur les données originales ${ }^{4}$

\subsection{Reconnaître que les limites ne sont pas systématiques}

L'apprenant, pour le moins déstabilisé par les énoncés qu'il rencontre, risque fort de surgénéraliser des prescriptions, conseils ou avertissements qu'il prend pour règles absolues et définitives. Il n'est que de citer quelques « règles de disposition» qu'un apprenant peut

\footnotetext{
2 Ce n'est pas un normal film sur les médecins. Enoncé extrait d'un corpus oral d'apprenants allophones, apprenant niveau B1.

${ }^{3}$ Ils vivent dans des cases petites. Enoncé extrait d'un corpus oral d'apprenants allophones, apprenant niveau B2.

${ }^{4}$ Extrait d'un mémoire de Master de «Modélisation, Ingénierie mathématiques » rédigé par un étudiant allophone.
} 
trouver dans des méthodes, manuels ou sites FLE. Ainsi, Geneviève-Dominique de Salins (2004 : 108) écrit : «[Les adjectifs habituellement placés devant le nom] sont peu nombreux mais leur emploi est très fréquent : petit - grand - jeune - vieux - gros - gentil - beau - joli bon - long - mauvais - etc. ». L'apprenant tentera de suivre cette règle envers et contre tout. Aussi pensera-t-il totalement juste un énoncé comme un gris ciel, d'autant qu'il aura également pu lire une autre règle de disposition stipulant la tendance à l'antéposition des adjectifs courts dans Sylvie Poisson-Quinton et al. (2002) : « Sont généralement placés avant le nom : quelques adjectifs courts, qui peuvent avoir plusieurs sens et sont donc très fréquents. Exemple: une belle histoire, un beau garçon, une belle âme, un bel automne, une belle sottise, un beau discours... ». Bien que gris soit fréquent et court, il lui faudra bien vite déchanter et accepter une autre règle spécifique pour classer les adjectifs de couleur : "Les adjectifs désignant la nationalité, les formes géométriques et les couleurs sont toujours placés après le nom : Une décision gouvernementale allemande. Des élections législatives italiennes. Une découverte scientifique française. » (de Salins 2004). Et une fois repéré que l'adjectif de couleur était postposé, comment va-t-il faire pour le coordonner avec l'adjectif petit généralement antéposé, s'il systématise ces dispositions ? Il risquera de produire un énoncé tel que celui présenté en (9).

9) ?les petit yeux et noirs ${ }^{5}$

Dans le même ordre d'idées, il aura pu lire : «si nom et adjectif sont tous les deux monosyllabiques, l'adjectif vient en général après le nom : un pas lent, un lit dur, la vie chère » (Poisson-Quinton et al. 2002). Règle que contrediront des énoncés comme le gros sel, le bon pain, le long cou.

L'apprenant devra donc très vite apprendre qu'aucune règle n'est absolue.

\subsection{Bilan : Un véritable problème pour l'enseignement}

Toute tentative de classification en fonction d'un seul critère et de façon catégorique est vouée à l'échec et les enseignants comme les concepteurs de manuels ou de méthodes tentent de conjurer cet échec de diverses façons qui, toutes, traduisent le malaise qu'ils ont à surmonter. En témoignent quelques exemples pris dans des ouvrages destinés à des apprenants non francophones. On y trouve des discours péremptoires, comme dans la citation 1, qui alternent avec des formulations embarrassées comme dans la citation 2, sans parler des inventaires à la Prévert :

\section{Citation}

Ces adjectifs [exprimant la nationalité, la forme, la religion, la couleur] ne sont jamais placés avant le nom (sauf, en poésie, les adjectifs de couleur).

(Monnerie-Goarin/Bréant 1988: 47)

\section{Citation}

D'autres [adjectifs] enfin, sont placés tantôt avant le nom, tantôt après, sans qu'il soit possible de trouver une règle bien précise.

(Monnerie-Goarin/Bréant 1988: 48)

\footnotetext{
${ }^{5}$ Elle a eu les cheveux noirs et longs, les petit yeux et noirs, les sourcils bien dessiné, et le nez retroussé. Extrait d'un corpus écrit, apprenant niveau B1.
} 
Exploiter un travail de corpus sur la place de l'épithète pour élaborer une progression didactique en FLE

Et même si certaines descriptions reconnaissent l'impasse des classifications comme Sylvie Poisson-Quinton et al. (2002: 98) qui l'exposent sans détour dans l'extrait présenté dans la citation 3, il apparaît nécessaire de se demander pourquoi ces tentatives de classement échouent.

\section{Citation}

[La place de l'adjectif épithète] est l'une des grandes difficultés du français car l'adjectif épithète peut être placé avant, après, ou indifféremment avant ou après le nom, en fonction d'un certain nombre de critères (longueur de l'adjectif, sens abstrait ou concret, etc.). Les règles sont complexes et, avouons-le, assez souvent floues. Nous allons cependant essayer d'en dégager quelques-unes.

(Poisson-Quinton et al. 2002: 98)

\section{$3 \quad$ Apport d'un travail sur corpus}

Le travail sur corpus mené dans la thèse de Thuilier (2012) permet, nous semble-t-il, de répondre au malaise des concepteurs de manuels et de dissiper le «flou » sur la disposition des adjectifs :

1. en démontrant l'absence de contraintes catégoriques,

2. en posant la notion de contraintes préférentielles,

3. en définissant les limites de ces contraintes,

4. en intégrant des paramètres syntaxiques, au-delà des propriétés de l'adjectif et du nom.

\subsection{Affirmation de l'absence de contraintes catégoriques}

Si toutes les tentatives de classement n'aboutissent jamais à des ensembles homogènes qui valident des règles catégoriques, c'est parce qu'il n'existe qu'une seule contrainte catégorique qui détermine la disposition des termes : la présence d'un dépendant adjectival à droite de l'adjectif impose la postposition du syntagme adjectival, comme cela est montré dans les exemples suivants.

10) une décision lourde / une lourde décision

11) une décision lourde de conséquences

12) *une lourde de conséquences décision

Au-delà des contre-exemples évidents aux quelques «règles » exposées dans la section 3 , le travail de Thuilier (2012: 104-136) présente des contre-exemples pour chacune des contraintes présentées dans la littérature, ce qui permet de démontrer que la question de la disposition n'est pas une question de normes, de distributions complémentaires mais une question d'usage.

\subsection{Contraintes préférentielles}

Bien que la notion de préférence soit sous-jacente dans les descriptions classiques et les manuels, elle n'apparaît que de façon particulièrement vague sous les termes de généralement ou habituellement, comme dans les extraits suivants :

\section{Citation}


[Les adjectifs ]sont généralement placés après le nom.

(Poisson-Quinton et al. 2002)

\section{Citation}

Quelques adjectifs très fréquents sont généralement antéposés.

(Chalaron/Abry 1996)

\section{Citation}

Les adjectifs [sont] habituellement placés devant le nom.

(Salins 2004)

La contrainte préférentielle peut être définie en ces termes : une contrainte préférentielle agit de façon non systématique sur le choix entre plusieurs réalisations possibles. Une contrainte est dite préférentielle lorsque sa présence est corrélée à une fréquence statistiquement significative d'une réalisation. L'absence de ce concept de «contrainte préférentielle » dans les manuels de FLE est explicable par son émergence récente qui n'a été rendue possible que par le renouveau technologique qui date des années 90 et la constitution de grands corpus richement annotés. À partir du traitement statistique d'un corpus, il est déjà possible de rendre visible l'impact des variables pouvant influer sur la position de l'adjectif et de les situer sur une échelle préférentielle. Nous ne retiendrons ici que quelques critères extraits des résultats de Thuilier (2012) dont le travail s'appuie sur les données extraites du corpus French Treebank. Ce corpus contient un ensemble d'articles du quotidien Le Monde datant de 1989 à 1995. Il est annoté pour les parties du discours, la flexion, la structure syntaxique et les fonctions grammaticales (cf. Abeillé/Barrier 2004; Abeillé/Clément 2003). Il contient 12351 phrases et 385458 tokens.

Premièrement, comme le montrent les données présentées dans le tableau 1, les adjectifs de plus de deux syllabes sont postposés dans leur grande majorité (92,6\%). Aussi sera-t-il intéressant de les présenter contrairement à ceux de moins de deux syllabes dont les données de corpus montrent qu'ils sont antéposés à $73,3 \%$ seulement. De même, les adjectifs bisyllabiques que l'on rencontre en postposition dans $63,9 \%$ des cas présentent une disposition plus aléatoire.

\begin{tabular}{|l|l|l|l|}
\hline & $\begin{array}{l}\text { Adjectifs longs } \\
\text { (plus de 2 syllabes) }\end{array}$ & $\begin{array}{l}\text { Adjectifs courts } \\
\text { (1 syllabe) }\end{array}$ & $\begin{array}{l}\text { Adjectifs de 2 } \\
\text { syllabes }\end{array}$ \\
\hline antéposé & & $73,3 \%$ & \\
\hline postposé & $92,3 \%$ & & $63,9 \%$ \\
\hline
\end{tabular}

Tableau 1 : Longueur de l'adjectif et position d'après les données de Thuilier (2012: 143)

La même remarque est valable pour la question de la fréquence : les données de corpus, synthétisées dans le tableau 2, montrent que la grande fréquence des adjectifs ne détermine pas forcément leur antéposition, alors qu'on peut s'appuyer sur leur rareté pour anticiper la postposition.

\begin{tabular}{|c|c|c|c|c|}
\hline & $\begin{array}{l}\text { Très } \\
\text { fréquents } \\
\text { (28 adjectifs) }\end{array}$ & \begin{tabular}{|l|} 
Assez \\
fréquents \\
(112 adjectifs)
\end{tabular} & \begin{tabular}{|l|} 
Moins \\
fréquents \\
(329 adjectifs)
\end{tabular} & $\begin{array}{l}\text { Rares } \\
\text { (1288 adjectifs) }\end{array}$ \\
\hline
\end{tabular}


Exploiter un travail de corpus sur la place de l'épithète pour élaborer une progression didactique en FLE

\begin{tabular}{|l|l|l|l|l|}
\hline antéposé & $66,7 \%$ & & & \\
\hline postposé & & $73,5 \%$ & $88,4 \%$ & $97,5 \%$ \\
\hline
\end{tabular}

Tableau 2 : Fréquence de l'adjectif et position d'après les données de Thuilier (2012: 148)

Les énoncés suivants, extraits du French Treebank, illustrent chaque catégorie de fréquence :

13) Très fréquent : Ces opérations [...] doivent permettre [...] d'accroître leurs parts de marché par de nouveaux axes de développement.

14) Assez fréquent : [...] la Pologne traverse une période difficile dans l'histoire de sa conscience morale $[\ldots]$

15) Moins fréquent : Dans un communiqué diffusé lundi matin par son canal habituel à Ajaccio, l'ex-FLNC a revendiqué l'attentat.

16) Rare : [...] le quotidien [...] a fait de M. Deng Xiaoping son homme de l'année [...] en dépit d'un aspect politiquement incorrect dû à son action répressive.

Les données de Thuilier (2012) attestent également du fait que les adjectifs morphologiquement construits sont massivement postposés $(93,7 \%)$ alors que les simples favorisent plutôt l'antéposition (50,7\%).

\begin{tabular}{|l|l|l|}
\hline & Adjectifs construits & Adjectifs simples \\
\hline antéposé & & $50,7 \%$ \\
\hline postposé & $93,7 \%$ & \\
\hline
\end{tabular}

Tableau 3 : Construction morphologique des adjectifs et position d'après les données de Thuilier (2012: 150)

Les énoncés suivants, extraits du corpus French Treebank, illustrent cette différence de prévisibilité de la disposition :

\section{Adjectifs construits}

17) Pour Matra Communication, les propositions européennes alléchantes n'ont pourtant pas manqué.

18) $[\ldots]$ les interventions [...] sont légitimes, d'autant plus que l'évolution technologique tend à réduire les solidarités naturelles.

\section{Adjectifs simples}

19) Le Royaume-Uni bénéficie d'une vive reprise après une récession profonde.

20) [...] le paysan paie le droit de cultiver une maigre parcelle en journées de travail sur les domaines des propriétaires.

En définitive, l'intérêt de la notion de contrainte préférentielle est qu'elle permet de rendre compte de la fréquence de certaines dispositions comme de la possibilité toujours ouverte de choisir une position non préférée.

\subsection{Définir les limites de chaque contrainte}

Le travail sur corpus vérifie et évalue la pertinence des paramètres retenus par les descriptions classiques pour le rôle qu'ils jouent dans la préférence de telle ou telle disposition. Il s'agit de 
prendre en compte les éventuels effets combinés des paramètres énoncés et de quantifier à l'aide de travaux sur corpus les effets de chaque paramètre. Ce travail sur les effets combinés des paramètres permet ainsi de mettre en évidence: (a) des contraintes illusoires, (b) des paramètres qui se répondent, (c) des contraintes susceptibles d'en neutraliser d'autres. Nous détaillons ces trois aspects dans la suite de cette section.

\section{(a) Des contraintes illusoires}

L'étude peut amener à invalider l'un des paramètres évoqués par la littérature en démontrant qu'il s'agit d'un effet second d'un autre paramètre. Ainsi la longueur relative de l'adjectif par rapport au nom est-elle un effet second de la longueur absolue de l'adjectif, qui, elle, est un véritable paramètre : « la longueur du nom n'intervient pas dans le choix de la position. La différence de longueur entre l'adjectif et le nom n'est significative que grâce à l'effet de la longueur de l'adjectif. La longueur relative n'est donc pas pertinente. » (Thuilier 2012 : 144). Ainsi, dans un jour lumineux, ce n'est pas le fait que l'adjectif lumineux soit plus long que le nom jour qui explique sa disposition, mais le seul fait qu'il soit long (3 syllabes). D'ailleurs, on trouve également un phénomène lumineux et un instrument lumineux.

\section{(b) Des contraintes qui se répondent}

La brièveté et la fréquence des adjectifs ne sont pas sans rapport, et ce, pour des raisons d'histoire de la langue, même si le travail de Thuilier (2012) montre qu'on ne peut pas les remplacer l'un par l'autre (à la différence de ce que propose Marc Wilmet (1981)).

Ainsi les adjectifs petit, grand, gros, court, lourd, bon, jeune, chaud, froid, beau sont-ils à la fois fréquents et courts. La plupart d'entre eux se rencontrent en antéposition mais pas tous (froid, chaud), sans oublier les adjectifs de couleur courts (gris, rose, vert, bleu, rouge, jaune, blanc, noir...) dont le corpus confirme la postposition à 100\%. Pour autant, certains termes fréquents ne sont pas courts (mauvais, excellent, rapide, sérieux, dangereux, tranquille, étrange, génial), ce qui interdit de remplacer les deux contraintes l'une par l'autre. De même, la construction d'un adjectif va logiquement de pair avec une certaine longueur (grand/agrandi, large/extra-large, doux/aigre-doux), ce qui diminue l'impact du paramètre « morphologiquement construit ».

\section{(c) Des contraintes susceptibles d'en neutraliser d'autres}

Certaines collocations peuvent invalider la préférence d'un adjectif pour une position. On entend par collocation, la fréquence très importante de co-présence d'un nom et d'un adjectif. Cette tendance au figement joue sur la disposition. C'est le cas, entre autres, de vibrant, qui a une préférence marquée en tant qu'adjectif verbal pour la postposition, préférence qui s'inverse dans la collocation vibrant hommage. Il en est de même pour immédiat après-guerre, vain mot, lourd tribut...

\subsection{Intégration des paramètres syntaxiques}

L'étude de Thuilier (2012) permet de repérer d'autres influences, en intégrant des paramètres syntaxiques, au-delà des propriétés de l'adjectif et du nom. Il en va ainsi de certains paramètres syntaxiques qui neutralisent les contraintes préférentielles favorisant l'antéposition. Ainsi, la coordination d'adjectifs va rendre plus fréquente la postposition d'adjectifs généralement antéposés. Des adjectifs tels que grand ou beau, qui ont une très 
Exploiter un travail de corpus sur la place de l'épithète pour élaborer une progression didactique en FLE

forte préférence pour l'antéposition (21-22), pourront être postposés s'ils sont coordonnés (23).

21) une grande table / ?une table grande

22) une belle table / ?une table belle

23) une table grande et belle

La présence d'un adverbe favorise aussi légèrement la postposition. Ainsi un adjectif peu enclin à la postposition, tel que grand (cf. exemple 21), peut-il facilement être placé après le nom s'il est modifié par un adverbe, comme dans l'exemple suivant :

24) une table assez grande

Les résultats de la thèse de Thuilier qui prennent en compte les corrélations des paramètres influençant la position de l'adjectif épithète, se présentent sous la forme d'une modélisation mathématique qui permet de prédire de façon très fiable la position d'un adjectif épithète. Si cette formule mathématique n'est pas directement utilisable pour l'enseignement, il n'en reste pas moins que la démonstration qui l'accompagne permet, nous l'avons vu, de résoudre les hésitations et approximations qui prédominent dans les descriptions destinées aux apprenants allophones. Le concepteur peut se baser sur les analyses et les exemples de corpus pour déterminer une progression d'apprentissage efficace. C'est ce que nous allons tenter de faire dans la section suivante.

\section{$4 \quad$ Progression d'apprentissage}

L'essentiel d'une progression est qu'elle permette à l'apprenant de se créer une grammaire implicite qui ne compromette pas, a postériori, la compréhension de l'ensemble du système.

La difficulté spécifique que pose l'élaboration d'une progression sur la position de l'épithète est la nécessité de prendre en compte l'usage dans son étendue et sa complexité pour en rendre compte ; usage qui bien évidemment fait particulièrement défaut à l'apprenant de FLE. D'où le choix de présenter un échantillonnage représentatif de l'usage de façon à baliser la progression d'apprentissage tout en évitant les généralisations hâtives.

\subsection{Niveau A1}

L'objectif est de donner une vision synthétique du système, c'est-à-dire de faire prendre conscience de l'absence de contraintes catégoriques mise en évidence par le travail de Juliette Thuilier (2012), tout en jetant les prémisses d'analyse des contraintes préférentielles. Se pose immédiatement un dilemme pour l'enseignant : comment présenter la liberté de disposition des adjectifs épithètes sans que l'apprenant imagine que cette disposition est aléatoire? Inversement, comment présenter les contraintes préférentielles sans que l'apprenant les prenne pour des contraintes absolues? Il nous a semblé que la réponse tenait dans l' «imitation» de l'usage par le biais d'un simple repérage visuel. Nous proposons d'appliquer la méthode de l'Apprentissage Sur Corpus présentée par Alex Boulton et Henry Tyne (2014) et développée dans l'optique de l'apprentissage de la disposition des adjectifs épithètes. Le recours au travail sur corpus est d'une importance cruciale : tout en proposant des énoncés authentiques, il justifie scientifiquement la démarche. L'objectif est donc de présenter, à travers des données attestées extraites d'un corpus, la notion de choix 
préférentiels instaurés par l'usage. À travers une activité d'observation, l'apprenant repère la position d'adjectifs fréquents par rapport au nom et observe que ces adjectifs peuvent être antéposés ou postposés, mais qu'une position est préférée.

Je vais vous demander une petite signature.

Il faut tirer la porte, il y a une petite poignée.

Le ruisseau où il y a une espèce de petite cascade.

Et c'est un endroit assez petit en fait.

Quand je serai bien en forme, je ferai une petite fête.

Il faut qu'elle laisse un petit mot.

D'après votre observation, complétez l'énoncé suivant en choisissant la place de petit :

Ils avaient loué une ... chambre... près du grenier.

(corpus Clapi ${ }^{6}$ )

\subsection{Niveau A2}

À ce niveau, il s'agit de commencer à repérer les critères de choix en distinguant d'abord quelques contraintes préférentielles fortes. Ainsi, à partir d'un corpus, les apprenants repèrent les catégories sémantiques pour lesquels la préférence de postposition est massive (couleur, forme, nationalité) en les comparant aux adjectifs déjà étudiés.

Le second objectif consiste à analyser le critère de longueur de l'adjectif. Il s'agit de repérer la postposition massive de l'adjectif long (plus de 2 syllabes) (92,3\% selon le corpus French TreeBank) tout en faisant comprendre qu'on ne peut pas s'appuyer sur l'idée inverse : l'antéposition des adjectifs épithètes courts. On présente un mini-corpus dans lequel tous les adjectifs sont postposés et longs sauf un court, ce dernier constitue l'intrus à repérer. L'observation permet à la fois de repérer la très forte tendance à la postposition des adjectifs longs et le fait que la réciproque n'est pas vraie.

\section{Quel est l'intrus?}

Il devait passer des analyses médicales, c'était une consultation nécessaire pour l'obtention de sa carte universitaire. Il arriva devant le centre hospitalier, un bâtiment imposant aux couloirs innombrables. Dans la salle d'attente aux murs froids, il y avait un nombre impressionnant d'étudiants.

Ce niveau peut également être l'occasion d'introduire la notion de collocation. En effet, une collocation, dans la mesure où elle présente un certain degré de figement, peut contredire une préférence de disposition forte (un vibrant hommage vs une consonne vibrante) ou faible (du temps libre vs un libre accès/un accès libre). Il est donc nécessaire que les apprenants prennent conscience de cette notion de collocation et qu'ils se construisent peu à peu une compétence lexicale.

\subsection{Niveau B1}

Le premier objectif à ce niveau est de poursuivre la présentation des contraintes fortes par l'introduction des adjectifs morphologiquement construits (postposés à 93,7 \% selon le corpus

\footnotetext{
${ }^{6}$ Corpus Clapi consultable en ligne : http://clapi.ish-lyon.cnrs.fr/V3_Accueil_Corpus.php?interface_langue=FR [20.06.2015].
} 
Exploiter un travail de corpus sur la place de l'épithète pour élaborer une progression didactique en FLE

French Treebank). La présentation se fait à partir du vocabulaire acquis à travers un réseau lexical. On présente la place de synonymes construits d'adjectifs déjà connus. Ainsi, beau dont l'antéposition a déjà été étudiée, sera-t-il associé à ses synonymes morphologiquement construits et donc préférentiellement postposés (séduisant, agréable).

Le deuxième objectif est d'examiner les conséquences de la coordination de l'adjectif et de la présence d'un adverbe avant l'adjectif. Comme l'a montré le travail sur corpus de Juliette Thuilier, ces constructions peuvent neutraliser une contrainte préférentielle. Cette neutralisation peut être exposée en complétant les corpus présentés en A1 par des énoncés où l'épithète n'apparaît pas seul mais est soit coordonnée à un autre épithète, soit modifié par un adverbe.

Il nous fallait venir dans cette maison petite et grise d'un village perdu.

... le menton petit et rond, un reste d'enfance curieux

... les confessions de nos vedettes du petit et grand écran, de nos écrivaines renommées.

Mon père est un géant petit et maigre dont l'air perdu inspire la pitié.

Corpus Frantext ${ }^{7}$

Je me vois dans mon pigeonnier trop petit, mal chauffé

il s'acharna à écrire sur un bout de papier trop petit une phrase trop grande

Mais je leur reproche à la fois leur trop petit nombre et leur modèle romanesque

J'ai du trop petit calibre, on ne fait rien avec ça.

Corpus Frantext

\subsection{Niveau B2}

C'est au niveau B2 qu'est introduite la question de la polysémie des adjectifs. L'objectif est alors de repérer qu'à l'opposition de disposition d'un même adjectif peut être associée une différence de sens du syntagme. Un corpus avec un terme déjà vu comme grand permet de mettre en évidence la polysémie de cet adjectif, polysémie qui exploite la disposition.

Il est aussi possible de créer des mini-corpus avec des paires minimales ${ }^{8}$ pour introduire une réflexion sur la polysémie des adjectifs, comme le proposent Camussi-Ni et Coatéval (2013):

\section{Vers la maîtrise des cas de contrainte de disposition des unités}

\section{- Les adjectifs polysémiques}

Ils sont postposés ou antéposés à l'autre unité du syntagme en fonction de la valeur sémantique sélectionnée :

- Est-ce que quelqu'un est venu visiter la maison?

- Il y a une personne seule qui est venue. Elle s'est étonnée qu'il n'y ait qu'une seule salle d'eau.

Disposition des unités et polysémie

\section{- Réflexion sur les conditions de sélection d'une des valeurs sémantiques de l'adjectif}

\footnotetext{
${ }^{7}$ Corpus Frantext consultable en ligne : http://www.frantext.fr [20.06.2015].

${ }^{8}$ L'expression «paire minimale » traditionnellement réservée à une opposition phonologique pertinente est ici étendue à la morpho-syntaxe. Il s'agit de faire observer la pertinence sémantique d'une variation syntaxique minimale entre deux énoncés.
} 
- Elles jouaient les grandes dames, tu vois le genre, elles pensaient que dans une grande maison, c'était nécessaire. En fait, c'étaient des femmes très communes.

- Et en plus, notre maison est petite.

Mise en évidence de cas de lexicalisation du syntagme

Alors qu'une grande dame s'oppose sémantiquement à une dame grande, il n'en est pas de même d'une grande maison qui n'active pas la polysémie du terme grande.

\subsection{Niveau C1}

La question de la polysémie peut être un objet d'étude très intéressant pour des étudiants de niveau avancé. On pourra proposer différents corpus sur quelques adjectifs pour favoriser l'analyse des jeux divers de polysémie. Par exemple, le corpus suivant permettra à l'apprenant de repérer des indices qui participent à la désignation soit de la «propriété » soit de l'antonyme de « sale ».

J'apprends ainsi, à ma propre surprise, que je parle anglais, mais que je ne le comprends pas.

Elle reconnaît ses propres traces dans le sable.

Elle prêchait la perte de tout intérêt propre, de la volonté, et même de la réflexion ;

Six personnages-fonctions, six éléments d'une machine dont chacun a sa fonction propre.

Il $\mathrm{y}$ a des fois où mes propres intuitions me terrifient.

Edmond, la conscience tranquille dans sa chemise propre, stationnait devant la maison.

Il traversait le carrelage propre du couloir.

Il était enfin, maintenant, à l'ombre ; assis au frais dans une petite chambre propre, que l'orientation vers le Nord protégeait hermétiquement du soleil.

Corpus Frantext

Chaque adjectif polysémique demandera alors une analyse particulière. Il s'agit de donner la capacité à utiliser des catégories déjà maîtrisées (+/- abstrait, +/- temporel, +/- espace, +/humain, +/- animé...) pour analyser le jeu de disposition de l'adjectif épithète liée à la polysémie.

À ce niveau, on pourra également se donner pour objectif de faire réfléchir à la possibilité d'aller contre l'usage pour créer des significations nouvelles ou à tout le moins de la nouveauté.

Un choix de disposition qui va à l'encontre des contraintes préférentielles faibles permet en effet d'attirer l'attention. Cette démarche est fréquente dans les corpus journalistiques, comme le montrent les exemples suivants :

Face à cette offre insuffisante, la demande est disproportionnée, quoique mal connue en raison des imprécisions des fichiers: leur simple cumul fait apparaître 300000 demandes en 1991, dont 100000 prioritaires, mais l'organisme, qui réclame depuis des années un observatoire régional des besoins et de la demande, estime que « le chiffre vrai doit se situer en-dessous, aux environs de $200000 »$

Ainsi, le certificat d'investissement Crédit Lyonnais continue à faire les frais de la coûteuse stratégie d'expansion de la banque et perd $7 \%$.

Elles restaurent ainsi leurs gains de productivité, leur situation financière et leur capacité d'investissement future. 
Exploiter un travail de corpus sur la place de l'épithète pour élaborer une progression didactique en FLE

Corpus French Treebank (Le Monde)

Apparait, ce qu'on pourrait nommer un effet de style dans ces écrits journalistiques, qu'il est intéressant de repérer.

La transgression est beaucoup plus nette quand la disposition va à l'encontre de contraintes préférentielles fortes comme dans les énoncés suivants :

Pour embrasser son grand-père, Aude se haussa sur la pointe de ses sandales de cuir qu'une ronde courroie retenait aux chevilles nues. (Albert Cohen, Solal)

Mais bientôt apparut une figure de nabot habillée d'une tunique à paillettes d'or et coiffée d'un bonnet à grelot d'argent, dont la main balançait un rouge lumignon dans les losanges vitrés d'une lanterne. (Aloysius Bertand, Gaspard de la nuit).

La très britannique banque d'affaire et de marché vient de...

Corpus Frantext

Corpus French Treebank (Le Monde)

Ou dans ceux-là qui transgressent le figement de disposition induit par les collocations :

Renverse, ô scribe, sur la table des grèves, du revers de ton style la cire empreinte du mot vain. (Saint John Perse, Exil)

Je la voyais, toute petite, dans le jardin de cette villa de highland park qui avait été l'occasion du mortel accident... et toujours, à un moment, la tragique question de la mourante retentissait distinctement à mes oreilles : « qu'expions-nous? » (Paul Bourget, Nos actes nous suivent)

Corpus Frantext

À ce niveau, on peut proposer aux apprenants de s'inspirer de ces textes pour créer leurs propres transgressions de la disposition avec des listes de collocation, pour créer de nouveaux sens, une langue nouvelle...

\section{Conclusion}

L'intérêt spécifique de ce travail est d'évaluer dans quelle mesure le didacticien peut chercher réponse à des problèmes d'enseignement de la langue dans des travaux sur corpus. Ces travaux sont particulièrement pertinents pour présenter des faits de langue qui mettent en jeu des contraintes préférentielles.

Ainsi, dans le cadre de la relation épithète, à l'absence de contraintes syntaxiques catégoriques dans la disposition des termes, l'usage a substitué des contraintes préférentielles. L'analyse de corpus permet de décrire ces contraintes et donne au didacticien les moyens de concevoir un apprentissage progressif et exhaustif.

L'observation de corpus est une démarche encore peu exploitée dans l'enseignement du FLE, pourtant elle assure une démarche pédagogique singulièrement heuristique et pourrait se montrer particulièrement adaptée à l'enseignement du FOS, ce qui nécessiterait la création de corpus ciblés qui correspondent à ces besoins didactiques.

\section{Bibliographie}

Abeillé, Anne/Godard, Danièle (1999): «La position de l'adjectif épithète en français: le poids des mots ». Recherches linguistiques de Vincennes 28: 9-32. 
Abeillé, Anne/Clément, Lionel (2003): "Building a Treebank for French”. In: Abeillé, Anne (ed.): Treebanks: Building and Using Parsed Corpora. Dordrecht, Kluwer: 165-187.

Abeillé, Anne/Barrier, Nicolas (2004): "Enriching a French Treebank. In: Calzolari, Nicoletta et al. (eds.): Proceedings of Language Resources and Evaluation Conference. Lisbon.

Abry, Dominique/Chalaron, Marie-Laure (1996): La grammaire des premiers temps. 2e édition. Grenoble: Presses Universitaires de Grenoble.

Benzitoun, Christophe (2013): «Adjectifs épithètes alternants en français parlé : premiers résultats ». In: Sabio, Frédéric (ed.): TIPA. Travaux interdisciplinaires sur la parole et le langage 29. http://tipa.revues.org/585 [23.07.2016].

Boulton, Alex/Tyne, Henry (2014): Des documents authentiques aux corpus : démarches pour l'apprentissage des langues. Paris: Didier.

Camussi-Ni, Marie-Armelle/Coatéval, Annick (2013): Comprendre la grammaire. Une grammaire à l'épreuve de la didactique du FLE. Grenoble: Presses Universitaires de Grenoble.

Delbecque, Nicole (1990): "Word order as a reflexion of alternate conceptual construals in French and Spanish. Similarities and divergences in adjective position". In: Cognitive Linguistics 1/4: 349-416.

Forsgren, Mats (1999): La place de l'adjectif épithète en français contemporain, étude quantitative et sémantique. Stockholm: Almqvist/Wilksell.

Monnerie-Goarin, Annie/Bréant, Marie-Thérèse (1988): Le français au présent. Paris: Hatier/Didier.

Noailly, Michèle (1999): L'adjectif en français. Paris: Ophrys.

Nølke, Henning (1980): «Où placer l'adjectif épithète ? Focalisation et modularité ». Langue française 111: 38-57.

Poisson-Quinton, Sylvie/Maheo-Le Coadic, Michèle/Mimran, Reine (2002): Grammaire expliquée du français. Paris: Clé International.

Reiner, Erwin (1968): La place de l'adjectif épithète en français : théories traditionnelles et essai de solution. Vienne/Stuttgart: Braumüller. (= Wiener romanistische Arbeiten 7).

Salins, Geneviève-Dominique de (2004): Grammaire pour l'enseignement du FLE. Paris: Didier.

Thuilier, Juliette (2012): Contraintes préférentielles et ordre des mots en français. Thèse de doctorat, Université Paris Diderot.

Thuilier, Juliette/Fox, Gwendoline/Crabbé, Benoît (2012): «Prédire la position des adjectifs épithètes en français : approche quantitative ». Lingvisticae Investigationes 35/1: 28-75.

Wilmet, Marc (1981): «La place de l'épithète qualificative en français contemporain : étude grammaticale et stylistique ». Revue de linguistique romane 45: 17-73. 